\title{
Corrigendum: ACE Gene Variants Rise the Risk of Severe COVID-19 in Patients With Hypertension, Dyslipidemia or Diabetes: A Spanish Pilot Study
}

\author{
María Íñiguez ${ }^{1}$, Patricia Pérez-Matute ${ }^{1}$, Pablo Villoslada-Blanco ${ }^{1}$, \\ Emma Recio-Fernandez ${ }^{1}$, Diana Ezquerro-Pérez ${ }^{2}$, Jorge Alba ${ }^{2}$, M. Lourdes Ferreira-Laso ${ }^{3}$ \\ and José A. Oteo ${ }^{1,2 *}$ \\ 1 Infectious Diseases, Microbiota and Metabolism Unit, Infectious Diseases Department, Center for Biomedical Research of \\ La Rioja (CIBIR), Logroño, Spain, ${ }^{2}$ Infectious Diseases Department, Hospital Universitario San Pedro, Logroño, Spain, \\ ${ }^{3}$ Department of Anesthesiology and Postoperative Care, Hospital Universitario San Pedro, Logroño, Spain
}

\section{OPEN ACCESS}

Edited and reviewed by: Hamad Ali,

Kuwait University, Kuwait

*Correspondence: José A. Oteo jaoteo@riojasalud.es

Specialty section: This article was submitted to Clinical Diabetes, a section of the journal

Frontiers in Endocrinology

Received: 06 September 2021 Accepted: 08 September 2021 Published: 20 September 2021

Citation:

Iñiguez $M$, Pérez-Matute $P$, Villoslada-Blanco $P$, Recio-Fernandez E, Ezquerro-Pérez D, Alba J, Ferreira-Laso ML and Oteo JA (2021) Corrigendum: ACE Gene Variants Rise the Risk of Severe

COVID-19 in Patients With

Hypertension, Dyslipidemia or Diabetes: A Spanish Pilot Study.

Front. Endocrinol. 12:771445. doi: 10.3389/fendo.2021.771445
Keywords: COVID-19, angiotensin converting enzyme, polymorphisms, hypertension, dyslipidemia, diabetes

\section{A Corrigendum on}

ACE Gene Variants Rise the Risk of Severe COVID-19 in Patients With Hypertension, Dyslipidemia or Diabetes: A Spanish Pilot Study

By Íñiguez M, Pérez-Matute P, Villoslada-Blanco P, Recio-Fernandez E, Ezquerro-Pérez D, Alba J, Ferreira-Laso ML and Oteo JA (2021). Front. Endocrinol. 12:688071. doi: 10.3389/fendo.2021.688071.

\section{ERROR IN FIGURE/TABLE}

In the original article, there was a mistake in Table 2 as published. Alleles of rs4343 polymorphism were incorrectly written. The corrected Table 2 appears below.

The authors apologize for this error and state that this does not change the scientific conclusions of the article in any way. The original article has been updated.

Publisher's Note: All claims expressed in this article are solely those of the authors and do not necessarily represent those of their affiliated organizations, or those of the publisher, the editors and the reviewers. Any product that may be evaluated in this article, or claim that may be made by its manufacturer, is not guaranteed or endorsed by the publisher.

Copyright (C) 2021 Íñiguez, Pérez-Matute, Villoslada-Blanco, Recio-Fernandez, Ezquerro-Pérez, Alba, Ferreira-Laso and Oteo. This is an open-access article distributed under the terms of the Creative Commons Attribution License (CC BY). The use, distribution or reproduction in other forums is permitted, provided the original author(s) and the copyright owner $(s)$ are credited and that the original publication in this journal is cited, in accordance with accepted academic practice. No use, distribution or reproduction is permitted which does not comply with these terms. 
TABLE 2 | Genotype frequencies of rs4341 and rs4343 polymorphisms in COVID-19 patients according to its hypertensive, dyslipidemic or diabetic status.

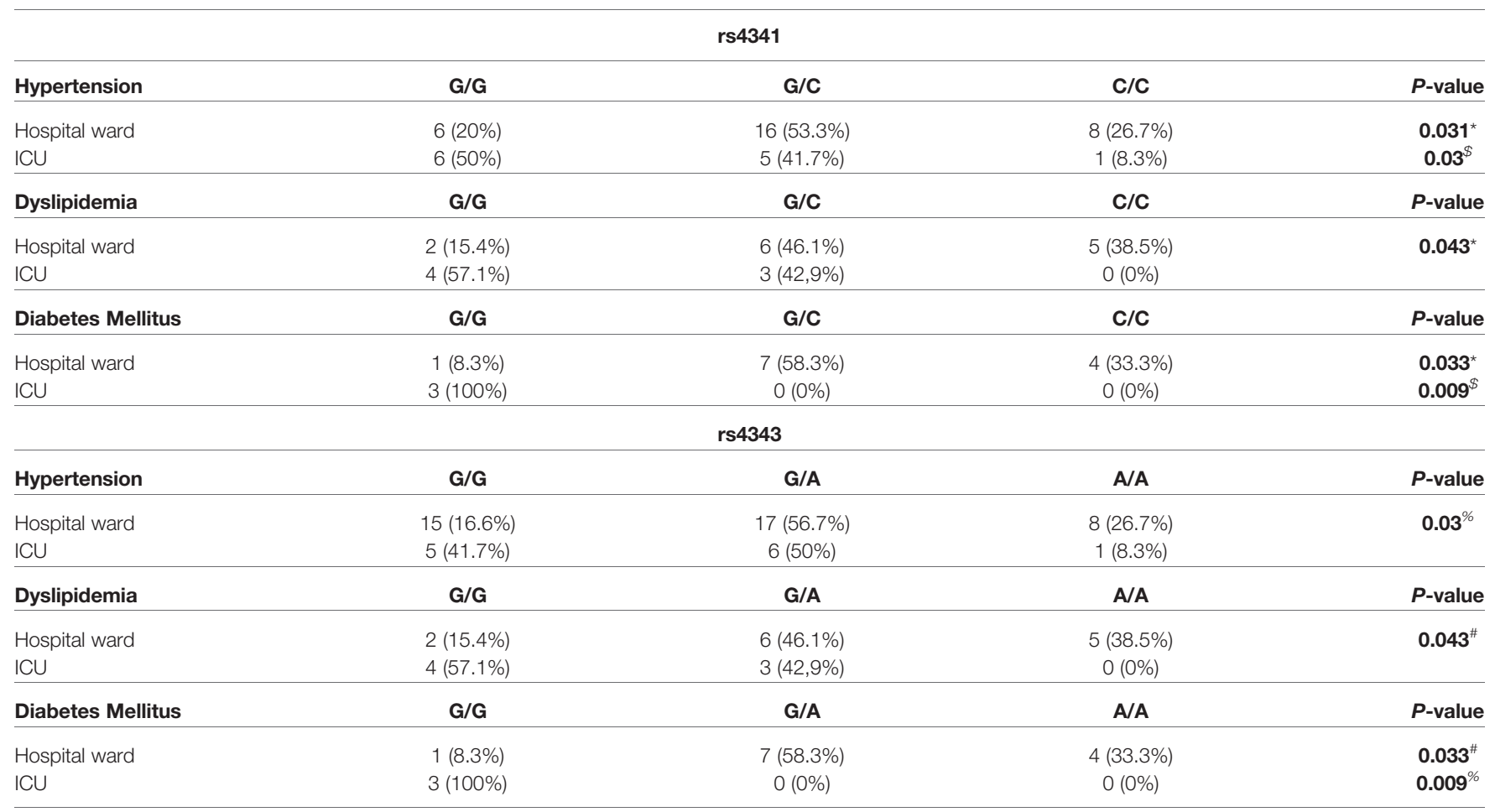

Data are presented as number of patients and percentages.

* $p$-value for group difference between GG and GC and CC.

${ }^{\$} p$-value for group difference between $G G+G C$ and $C C$.

${ }^{\#} p$-value for group difference between $G G+G A$ and $A A$.

${ }^{\%} p$-value for group difference between $G G+G A$ and $A A$.

Significant differences appear in bold case.

All data were adjusted for age, sex, BMI and number of comorbidities. 